\title{
Initial Experience with Contrast Enhanced Digital Mammography (SenoBright) - In a Comprehensive Clinical Breast Center
}

\author{
Robert L. Elliott, Catherine C. Baucom, Mary C. Elliott, Emorly H. Millet, Xianpeng Jiang \\ Elliott-Baucom Breast Cancer Research and Treatment Center, Baton Rouge, LA, USA \\ Email: jiang@eehbreastca.com
}

How to cite this paper: Elliott, R.L., Baucom, C.C., Elliott, M.C., Millet, E.H. and Jiang, X.P. (2017) Initial Experience with Contrast Enhanced Digital Mammography (SenoBright)-In a Comprehensive Clinical Breast Center. Journal of Cancer Therapy, 8, 146-154.

https://doi.org/10.4236/jct.2017.82012

Received: January 7, 2017

Accepted: February 7, 2017

Published: February 10, 2017

Copyright $\odot 2017$ by authors and Scientific Research Publishing Inc. This work is licensed under the Creative Commons Attribution International License (CC BY 4.0).

http://creativecommons.org/licenses/by/4.0/

\begin{abstract}
Objective: The purpose of initiating contrast enhanced digital mammography in our center was to evaluate the complimentary benefit of this technology with screening digital mammography and real time ultrasound in equivocal cases and high risk patients with dense breast. The intended goal was to reduce the incidence of further diagnostic and invasive procedures. Methods: Patients thought to be candidates who had good renal function confirmed by serum Blood Urea Nitrogen (BUN) and Creatinine were offered the procedure, and 225 patients had the procedure during the period of March 2013 through November 2014. The contrast enhanced digital mammograms (SenoBright) are performed on the Senograph Essential Unit. A total of 8 images are obtained: 4 conventional digital mammograms and 4 contrast enhanced digital mammograms. The patients with a positive SenoBright study had a tissue diagnosis of the lesion obtained by either a stereotactic needle biopsy, ultrasound guided core biopsy, or ultrasound directed open excisional biopsy. Results: The 225 patients who had the procedure included high risk patients with dense breast (41), patients with abnormal mammograms (92), and patients with equivocal clinical, mammographic and real time ultrasound findings (92). 31 studies were interpreted as positive and 194 as negative. 33 biopsies were performed, with 31 patients having a positive study and 2 patients with a negative study. 22 cancers were detected. Conclusion: We found that the addition of dynamic contrast enhanced digital subtraction mammography (SenoBright) was helpful in distinguishing malignant from non-malignant lesions. It was also effective in demonstrating multifocal lesions and identifying non-palpable occult carcinomas in the dense breast. It proved to be a valuable complimentary adjunctive diagnostic modality for a comprehensive clinical breast center.
\end{abstract}

\section{Keywords}

Breast Cancer, Mammography, SenoBright 


\section{Introduction}

Breast cancer is a very common female disease affecting 1 in 8 women in the United States. It is difficult to diagnose in the dense breast even with screening digital mammography. It has been reported that at least one in four malignant tumors are undetected with screening digital mammography [1]. As a clinical diagnostic breast center, we have been investigating new technology to add to our diagnostic armamentarium. This is important in the present health care environment, especially when insurance carriers are denying payment for dedicated breast MRI even in patients already diagnosed with breast cancer.

At this time, we must distinguish our comprehensive clinical breast center from just a breast imaging center. Patients who come to our center have a complete evaluation and do not have just a mammogram that will be read later by a radiologist. Our patients have a history and a thorough clinical breast examination by a physician and a screening digital mammogram. The mammogram is immediately read and interpreted while the patient is still present in the center. If there is an indication for ultrasound, it is done in real time by a physician; and it is not done by a technologist who presents hard copy data to be interpreted later by a radiologist. Therefore, the patient knows before the end of the visit if there is a problem that requires a biopsy or further diagnostic procedures. If the results indicate a need for biopsy, then either a stereotactic core needle or ultrasound directed core biopsy is done during that clinic visit. This avoids unnecessary recalls, thus eliminating severe patient anxiety. Many of the problems are minimal, and days or weeks of unnecessary patient anxiety are avoided. I have had the opportunity to see many patients who have had both types of experiences, and the one-stop evaluation of the center wins the patient evaluation every time.

For patients needing further diagnostic tests, the SenoBright procedure was attractive for several reasons: 1 . Patients are familiar with mammography; 2 . The procedure is inexpensive; 3 . SenoBright demonstrates anatomic and physiological information; 4. There is no delay in informing the patient of the results; 5 . The study is very easy to interpret; 6 . There are previous great studies from Europe and Canada [2]-[7].

\section{Methods}

The clinical protocol and informed consent form were reviewed by our institutional review board. All patients signed an informed consent form prior to enrollment to the protocol. Patients thought to be candidates for the SenoBright had blood drawn for BUN and creatinine levels to ensure good kidney function. Patients with good renal function (Creatinine 88 - $128 \mathrm{~mL} / \mathrm{min}$; BUN 10 - 20 $\mathrm{mg} / \mathrm{dL}$ ) are then scheduled for the procedure. The contrast enhanced digital mammograms (SenoBright) are performed on the GE Senograph Essential Unit equipped with a cesium iodide detector plate with molybdenum and rhodium filters. The SenoBright images are produced by low and high energy. The high energy images are produced by the filters in the detector plate changing to a 
copper filter combination of molybdenum or rhodium.

At the start of the procedure, the patient is seated in a chair to sign the consent and have a good IV started. The procedure is explained to the patient and all questions answered. The IV catheter is then connected to the dual pressure injector (Optivantage D H, Mallinckwelt) which delivers saline and contrast (Omnipaque 300) to the patient. The patient receives $1.5 \mathrm{ml}$ of Omnipaque 300 per kilogram body weight. The dual pressure injector system is set for a flow rate of $2.0 \mathrm{ml} / \mathrm{sec}$ with a 275 peak psi. $20 \mathrm{ml}$ of saline is given first with a $10 \mathrm{sec}$. delay to ensure no infiltration of the IV. A timer is then started and the contrast is administered followed by $20 \mathrm{ml}$ of saline. The first exposure is taken 2 minutes later. The unaffected breast is first to be compressed in both the craniocauded and mediolateral views after the injection. The low and high energy images are taken at the same time, as the machine automatically switches from molybdenum or rhodium combination to the copper filter combination depending on the density of the breast tissue. The affected breast is compressed for 4 minutes. A total of 8 images are obtained: 4 conventional digital mammograms and 4 contrast enhanced digital mammograms. The total time for the procedure is 7 minutes. It is important to obtain all images within 7 minutes to ensure optimal contrast up-take by the breast. After the procedure, the patient is taken to the work station to review the study with a physician. The results are discussed and the patient knows at that time if she has a problem. If a biopsy is indicated, it is usually done at that visit.

\section{Results}

As shown in Table 1, there were 225 total patients in the initial study. 41 patients with dense breast tissue, 92 patients with an abnormal mammogram, and 92 patients with equivocal findings on mammogram and/or ultrasound were included. There were 31 patients considered to have a positive SenoBright study out of 225 patients or $14 \%$. As shown in Table 1, 23 patients were from the abnormal mammogram group, 6 were from the equivocal findings group, and 2 were from the dense breast tissue group. As shown in Table 1, 22 patients (71\%) out of 31 positive SenoBright studies had pathologically proven breast malignancy. 18 patients (82\%) had invasive ductal carcinoma of the breast, and 4 patients (18\%) had ductal carcinoma in situ of the breast. There were 9 patients (29\%) out of the 31 positive SenoBright studies who had benign findings. However, 5 out of the 9 benign pathological findings (56\%) had a tissue diagnosis showing high risk proliferative changes such as atypical ductal hyperplasia and severe intraductal papillomatosis. The other 4 benign pathological findings revealed 3 proliferative fibroadenomas (33\%), while 1 was only bland, benign fibrocystic changes of the breast. There were 2 patients who had a negative SenoBright and who had a breast biopsy because of their mammogram findings or patients' request due to anxiety. Of these 2 patients with negative SenoBright, 1 patient had low grade ductal carcinoma in situ, while the other patient had a benign fibroadenoma (Table 1). 
Table 1. Clinical diagnostic criteria and pathological findings.

\begin{tabular}{|c|c|c|}
\hline & Criteria or findings & Number of patients \\
\hline \multirow{3}{*}{ All 225 patients } & Dense breast tissue & 41 \\
\hline & Abnormal Mammogram & 92 \\
\hline & $\begin{array}{l}\text { Equivocal findings on Mammogram or } \\
\text { Ultrasound }\end{array}$ & 92 \\
\hline \multirow{3}{*}{$\begin{array}{l}\text { Patients with Positive } \\
\text { SenoBright }\end{array}$} & Dense breast tissue & 2 \\
\hline & Abnormal Mammogram & 23 \\
\hline & $\begin{array}{l}\text { Equivocal findings on Mammogram or } \\
\text { Ultrasound }\end{array}$ & 6 \\
\hline \multirow{5}{*}{ Patients Biopsied } & Invasive Ductal Carcinoma of the breast & 18 \\
\hline & Ductal Carcinoma of the Breast & 4 \\
\hline & $\begin{array}{l}\text { High risk for breast cancer with proliferative } \\
\text { changes including atypical ductal hyperplasia } \\
\text { or papillomatosis }\end{array}$ & 5 \\
\hline & Proliferative fibroadenomas & 3 \\
\hline & Benign fibrocystic changes & 1 \\
\hline
\end{tabular}

\section{Discussion}

Duel-energy contrast enhanced digital mammography (CEDM) has been used in a number of centers in Europe for several years. However, the experience with this technology and procedure has been used infrequently in the United States. Dromain, Thibault, and Diekmann, et al. [8] from France have published an impressive paper on the procedure. They presented their initial clinical results of a multireader, multicase study. They concluded that (CDEM) as an adjunct to mammography and ultrasound (US) improved the diagnostic accuracy compared to mammography and (US) alone. They stated that the addition of iodinated contrast agent to digital mammography improves visualization of breast lesions.

Lewin and colleagues published one of the first preliminary clinical studies using the CEDM dual energy technique [7]. They examined 26 women with 12 benign and 14 malignant lesions prior to breast biopsy. Twelve of the 13 invasive carcinomas showed strong or moderate enhancement, while one demonstrated weak enhancement. Ten of the 12 benign lesions showed no enhancement, while the other 2 demonstrated very weak enhanced CEDM images. Evidence indicates that CEDM imaging improves the detection of suspicious areas and their differentiation by its ability to detect angiogenesis. The technology has the possibility to improve breast cancer detection, improve breast cancer staging, and improve selection of patients for biopsy. Its greatest advantage is that it adds physiologic information to the anatomic findings of mammography.

The disadvantage of this procedure is the risk of adverse side effects such as allergic reactions. Any center offering this procedure should have all personnel involved in the test trained and fully prepared to treat any adverse reaction. Emergency supplies and equipment should be present in the mammography suite. We have been blessed and fortunate to not have, so far, any serious adverse 
reactions.

CEDM allows for bilateral examinations with only one contrast injection and is very good for staging newly diagnosed breast cancers. We believe it can also be used for planning breast cancer conserving surgery, thus replacing MRI to rule out multicentric and multifocal disease. Earlier Dromain and colleagues published an article on the evaluation of tumor angiogenesis of breast carcinoma using CEDM [3]. They compared the CEDM findings with the histologic analysis of the surgical specimen. This analysis also included intratumoral microvessel density quantification studied on CD34 immunostained histologic tissue sections. They concluded that CEDM is able to detect angiogenesis in breast carcinoma but had poor correlation on data measured by CEDM and intratumoral-microvessel density measured by CD34 immunostained histologic sections [3].

In her paper on CEDM, Maxine Jochelson made several key points: 1) CEDM can improve the sensitivity of digital mammography; 2) CEDM is less sensitive but more specific than MR imaging; 3) CEDM is significantly less expensive than MRI and could potentially be used for screening patients who are unable to undergo breast MRI [9].

Jong, Yaffe and Skarpathiotakis, et al. [6] presented their initial clinical experience with CEDM and believed that CEDM could potentially be useful in identifying lesions in the mammographically dense breast. Their paper was published in 2003, and they suggested that further investigation is certainly warranted.

Sleeba and Subapradhe, et al., have reported on the role of CEDM as a problem-solving tool in the dense breast. They believe that a major clinical indication for CEDM is its use as a problem-solving technology in the case of equivocal mammographic and (US) findings [10]. We totally agree with their judgement, and have found CEDM to be extremely helpful in the localization of mammographic densities seen on only one mammographic view. If the lesion is of significance CEDM enhancement easily localizes the lesion on both views and aids greatly in planning the biopsy.

Another study recently compared dual energy CEDM and breast MRI. This study showed similar sensitivities but noted superior specificity for CEDM in the detection of primary breast cancer [11].

Our experience regarding MRI and CEDM has been similar, but CEDM has some advantages. CEDM is economical and can be easily available, avoiding long waiting times and delayed reports for MRI. The prevention of delay significantly reduces patient anxiety. CEDM is easy to perform, and it is much cheaper and much easier for the examiner to interpret. In fact, we have found that, after explaining the rationale of the technology to the patient while at the work station, they are able to detect the abnormality on the (SenoBright) images if one is present.

Akin, Brennan, and Dershow et al., have reported on advances in oncologic imaging and have described CEDM as an important emerging technology providing diagnostic accuracy approaching that of MRI [12]. CEDM has an advan- 
tage over tomosynthesis as there is much less radiation involved with the procedure, and it also provides physiologic as well as anatomic information. This physiologic information is valuable distinguishing benign from malignant lesions. A well-known mammographer who conducts mammography training seminars worldwide told me that tomosynthesis produces too much radiation and is for mammographers who do not know how to read mammograms and use real time (US)! Tomosynthesis as a 3-D mammogram is being used as a marketing tool.

In 2014, Fallenberg, Dromaine, Diekmann, and Renz et al. published a paper entitled "Contrast-Enhanced Spectral Mammography: Does Mammography Provide Additional Benefits or Can Some Radiation Exposure be Avoided [13]? They found that contrast enhanced spectral mammography (CESM) and the combination of CESM mammography (MG) to be superior to MG alone in tumor detection. There was a $16.8 \%$ increase in lesion detection using CESM compared to MG, and a $0.3 \%$ difference compared to CESM plus MG. The sensitivity was increased even more in premenopausal women and women with dense breast. Their study also showed that CSEM alone had the closest correlation with pathology. They also concluded that CSEM as an adjunct to MG had higher sensitivity and specificity compared to MG alone and also to MG in combination with ultrasound.

Our CDEM experience detected 22 malignancies in 31 patients considered to have a positive CEDM (SenoBright) procedure. There were 18 invasive ductal carcinomas and 4 ductal carcinomas in situ (DCIS). The invasive carcinomas demonstrated moderate to intense enhancement (Figure 1). The (DCIS) images showed less intense but definite enhancement. Nine other patients with benign disease had a positive study showing mild to less moderate intensity enhancement. Five of these 9 patients had high risk proliferative lesions, while 4 others had active proliferative fibroadenomas and one had benign fibrocystic changes. Two patients had a negative (SenoBright) procedure but had a biopsy because

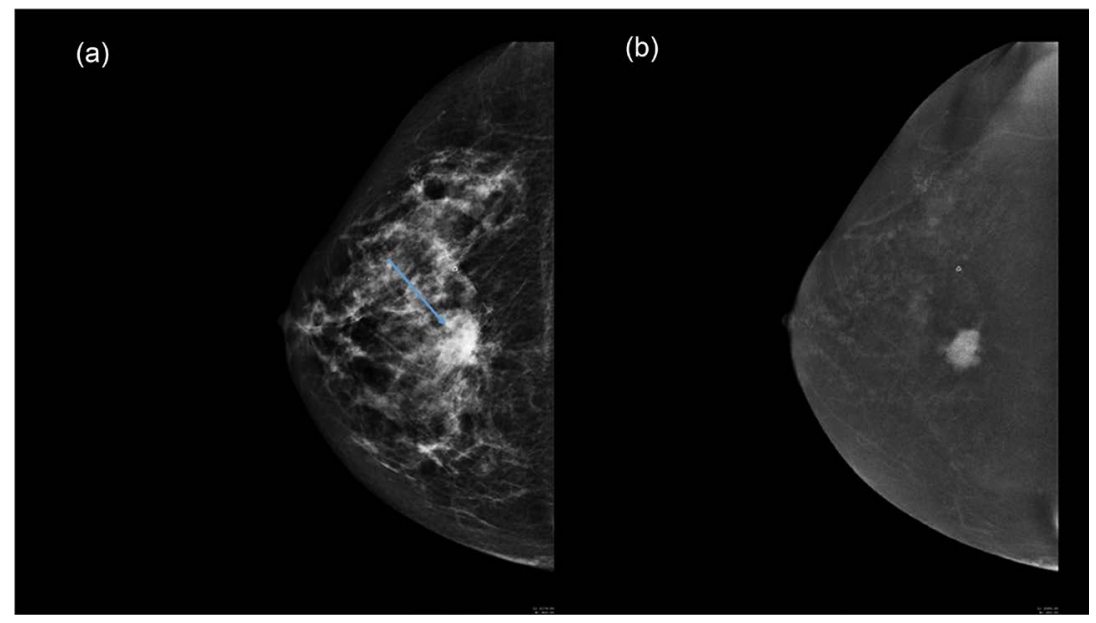

Figure 1. (a) Cranialcaudial view of digital mammogram right breast showing dense matrix discrepancy at (arrow). (b) SenoBright image of same area showing intense focal enhancement of the area. Biopsy revealed invasive ductal carcinoma. 
of patient anxiety and it was early in offering the procedure. One had very low grade (DCIS) and the other had a benign fibroadenoma (Figure 2).

We had two positive (SenoBright) patients with unique findings. One patient with suspicious mammographic calcifications in the left breast showed mild enhancement in that area and more intense enhancement distant from that area in the same breast. The area of the microcalcifications was (DCIS), while the unsuspected enhanced area was an invasive carcinoma. The other patient had a questionable lesion in the right breast on the mammogram. The (SenoBright) showed no enhancement in the right breast, but there was an unexpected area of enhancement in the contralateral left breast. This area proved to be invasive ductal carcinomas (Figure 3).

Another tremendous benefit of the SenoBright procedure is the detection of equivocal and occult simple and complex cyst. We call this reversed image enhancement, and to our knowledge, this has not been mentioned or emphasized in the literature. The phenomenon is created by the diffuse blush of the contrast throughout the breast parenchyma. The contrast does not enter into the cyst dead space that is filled with fluid and cellular debris. The result is a smooth well circumscribed image darker than the contrast blush in the surrounding breast stroma. This reversed imaging phenomenon has saved many patients from a surgical procedure (Figure 4).

\section{Conclusion}

We have found CEDM to be a tremendous and inexpensive adjunct to digital mammography and real time (US), especially in the dense breast and equivocal cases. The technology is comparable to MRI in cancer detection and in determining the size of the lesion and is better than MRI in detecting multifocal, multicentric, and bilateral disease. CEDM has less radiation than tomosynthesis and also provides angiogenic information. CEDM is also valuable in surgical planning and reduces the incidence of unnecessary breast biopsies. There has been

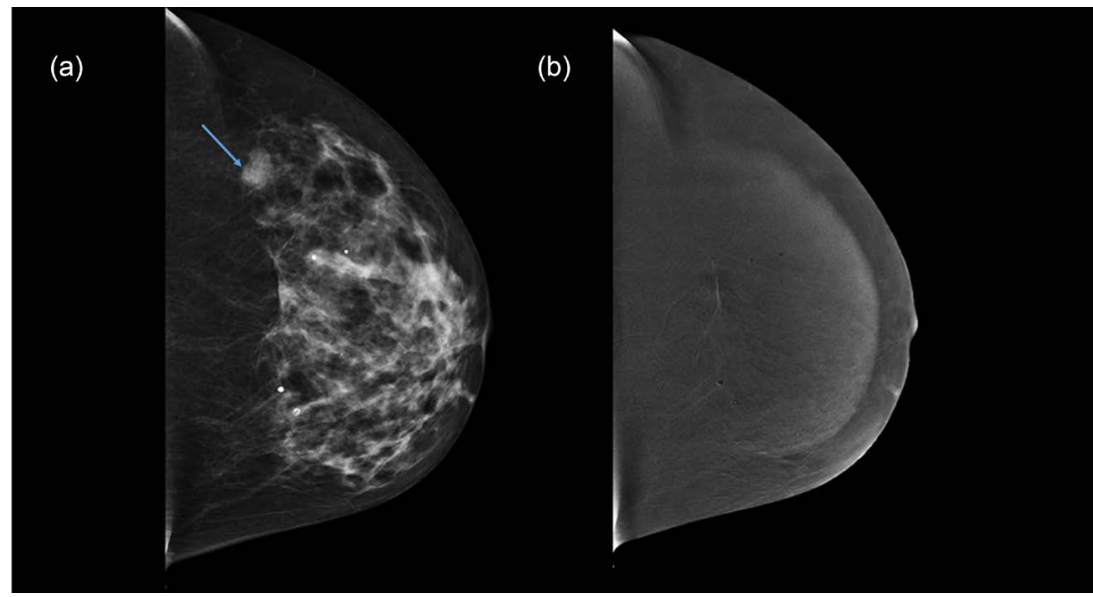

Figure 2. (a) Cranialcaudial view of digital mammogram left breast showing a well circumscribed smooth density (arrow). (b) SenoBright image of the same breast showing no contrast enhancement of that density. Biopsy was a benign fibroadenoma. 


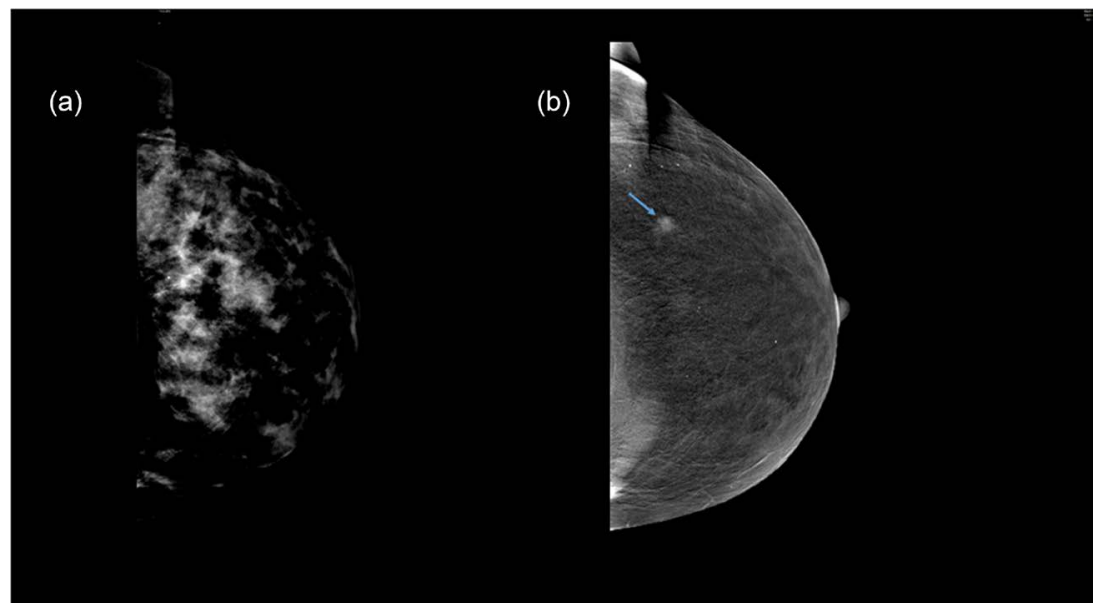

Figure 3. (a) Cranialcaudial view left breast of digital mammogram showing very dense breast with no major abnormalities. (b) SenoBright image of the same view showing a small area of intense focal enhancement. Biopsy proved to be an invasive ductal carcinoma.

(a)

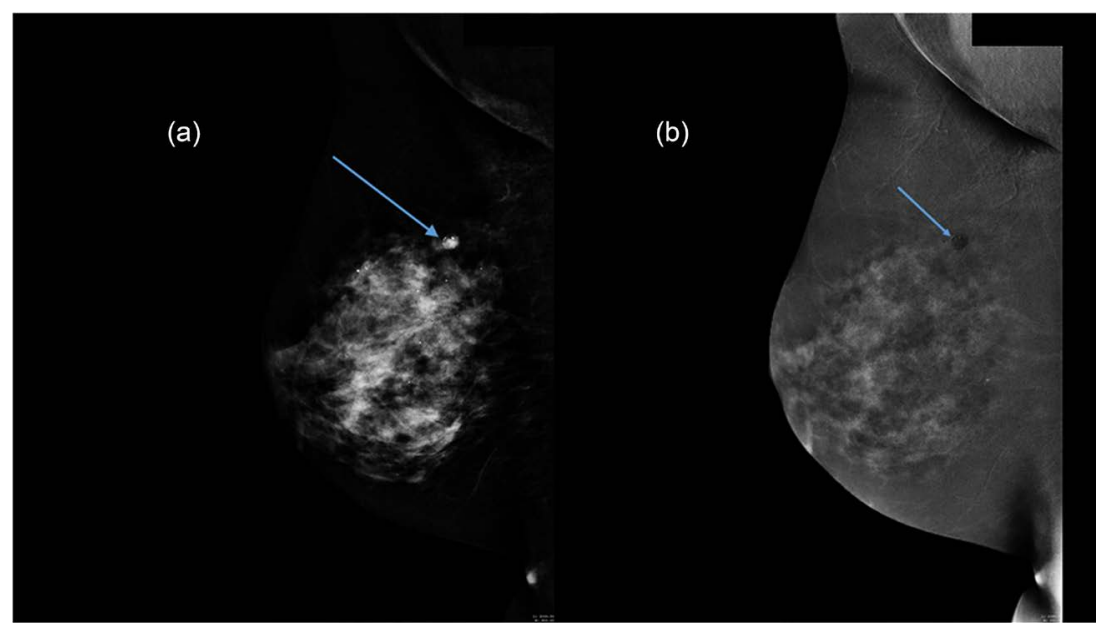

Figure 4. (a) Mediolateral view of digital mammogram right breast showing small round dense mass (arrow). (b) SenoBright image of the same breast showing a dark well-circumscribed area of reversed enhancement of the density (arrow). Confirms area is a benign fibrotic cyst.

great patient acceptance. CEDM is tremendous and should be part of the diagnostic armamentarium of any breast center, especially a clinical comprehensive breast center.

\section{Acknowledgements}

The authors are extremely grateful to Susie Billodeaux and Valerie Troiani for their valuable assistance in the preparation of this manuscript.

\section{References}

[1] Pisano, E.D., Gatsonis, C. and Hendrick, E. (2005) Diagnostic Performance of Digital versus Film Mammography for Breast-Cancer screening. The New England Journal of Medicine, 17, 1773-1783. https://doi.org/10.1056/NEJMoa052911 
[2] Dickmann, F., Diekmann, S. and Jeunehomme, F. (2005) Digital Mammography Using Iodine-Based Contrast Media "Initial Clinical Experience with Dynamic Contrast Medium Enhancement. Investigative Radiology, 40, 397-404. https://doi.org/10.1097/01.rli.0000167421.83203.4e

[3] Dromain, C., Balleyquier, C. and Muller, S. (2006) Evaluation of Tumor Angiogenesis of Breast Carcinoma Using Contrast-Enhanced Digital Mammography. AJR, 187, 528-537. https://doi.org/10.2214/AJR.05.1944

[4] Diekmann, F., Freyer, M., Diekmann, S. and Fallenberg, E.M. (2011) Evaluation of Contrast-Enhanced Digital Mammography. European Journal of Radiology, 78, 112-121. https://doi.org/10.1016/j.ejrad.2009.10.002

[5] Diekmann, F. and Ulrich, B. (2007) Tomosynthesis and Contrast-Enhanced Digital Mammography. European Radiology, 17, 3086-3092.

https://doi.org/10.1007/s00330-007-0715-x

[6] Jong, R.A., Yaffe, M.J. and Skarpathiotakis, M. (2003) Contrast-Enhanced Digital Mammography: Initial Clinical Experience. Radiology, 228, 842-850. https://doi.org/10.1148/radiol.2283020961

[7] Lewin, J.M., Isaacs, P.K., Vance, V. and Larke, F.J. (2003) Dual-Energy ContrastEnhanced Digital Subtraction Mammography: Feasibility. Radiology, 229, 261-268. https://doi.org/10.1148/radiol.2291021276

[8] Dromain, C., Thibault, F., Dickmann, F. and Fallenberg, E.M. (2012) Dual-Energy Contrast-Enhanced Digital Mammography: Initial Clinical Results of a Multireader, Multicase Study. Breast Cancer Research, 14, 1-17. https://doi.org/10.1186/bcr3210

[9] Jochelson, M. (2014) Contrast-Enhanced Digital Mammography. Radiologic Clinics of North America, 52, 609-616. https://doi.org/10.1016/j.rcl.2013.12.004

[10] Sleeba, T., Subapradha, A., Ramachondran, M. and Krishnoswami, M. (2013) Role of Dual-Energy Contrast-Enhanced Digital Mammography as a Problem-Solving Tool in Dense Breasts: A Case Report. Indian Journal of Radiology and Imaging, 23, 219-222. https://doi.org/10.4103/0971-3026.120269

[11] Jochelson, M.S., Dershaw, G.D., Sury, J.S. and Heendt, A.S. (2013) Bilateral Contrast-Enhanced Dual-Energy Digital Mammography: Feasibility and Comparison with Conventional Digital Mammography and MR Imaging in Women with Known Breast Carcinoma. Radiology, 266, 743-751.

[12] Akin, O., Brennan, S.B., Dershaw, D.D. and Ginsberg, M.S. (2012) Advances in Oncologic Imaging: Update on 5 Common Cancers. CA: A Cancer Journal for Clinicians, 62, 364-393. https://doi.org/10.3322/caac.21156

[13] Fallenberg, E.M., Dromaine, C., Diekmann, F. and Rezy, D.M. (2014) ContrastEnhanced Spectral Mammography: Does Mammography Provide Clinical Benefits or Can Some Radiation Exposure Be Avoided? Breast Cancer Research and Treatment, 146, 371-381. https://doi.org/10.1007/s10549-014-3023-6 
Submit or recommend next manuscript to SCIRP and we will provide best service for you:

Accepting pre-submission inquiries through Email, Facebook, LinkedIn, Twitter, etc. A wide selection of journals (inclusive of 9 subjects, more than 200 journals)

Providing 24-hour high-quality service

User-friendly online submission system

Fair and swift peer-review system

Efficient typesetting and proofreading procedure

Display of the result of downloads and visits, as well as the number of cited articles Maximum dissemination of your research work

Submit your manuscript at: http://papersubmission.scirp.org/

Or contact jet@scirp.org 